\title{
A MICRO-PULSE DifFERENTIAL ABSORPTION LIDAR TEST NETWORK
}

\author{
Scott Spuler ${ }^{* 1}$, Todd Bernatsky ${ }^{1}$, Catharine Bunn ${ }^{3}$, Joshua Carnes ${ }^{1}$, Matthew Hayman ${ }^{1}$, Kevin Repasky ${ }^{4}$, \\ Robert Stillwell ${ }^{2}$, Tammy Weckwerth ${ }^{1}$ \\ ${ }^{1}$ National Center for Atmospheric Research, Earth Observing Lab, Boulder, CO 80301, USA, *spuler@ucaredu \\ ${ }^{2}$ National Center for Atmospheric ResearchAdvanced Study Program, Boulder, CO 80301, USA \\ ${ }^{3}$ Montana State University, Physics Department, MT 59717, USA \\ ${ }^{4}$ Montana State University, Electrical and Computer Engineering, Bozeman, MT 59717, USA
}

\begin{abstract}
The National Center for Atmospheric Research (NCAR) and Montana State University (MSU) have developed a test network of five micro-pulse Differential Absorption Lidar (DIAL) instruments to continuously measure highvertical-resolution water vapor in the lower atmosphere. The instruments are accurate, low-cost, operate unattended, and eye-safe - all key features to enable larger 'national-scale' networks needed to characterize atmospheric moisture variability which influences important processes related to weather and climate.
\end{abstract}

\section{INTRODUCTION}

Water vapor is one of the fundamental thermodynamic variables that define the state of the atmosphere. It is highly variable in space and time and influences many important processes related to weather and climate. Several National Research Council (NRC) reports and a recent review paper, detailing the state of the art for thermodynamic profiling,- all highlight the need for improved water vapor measurements as a necessary step toward improving mesoscale numerical weather prediction and quantitative precipitation forecasting skills [1, 2, 3, 4]. To help address this observational need, we have developed a testbed of five diode-laser-based, micro-pulse DIAL (MPD) instruments for measuring the spatial and temporal distribution of water vapor in the lower atmosphere.

Developments in semiconductor laser and amplifier technology have enabled compact DIAL transmitters with narrow linewidths, and high spectral purity at low cost and with long lifetimes [5, 6, 7]. Semiconductor lasers are well suited to lidar systems designed for network deployment where instruments must be capable of unattended operation for long periods without maintenance and should not pose a risk to aircraft or bystanders. The network testbed described herein, was built from a fieldcapable semiconductor-based lidar prototype first demonstrated in 2015, which was shown to deliver accurate measurements of water vapor in the lower troposphere and produce scientifically significant data $[8,9]$.

\section{INSTRUMENT DESIGN}

The instrument, shown schematically in Figure 1, utilizes a diode-laser-based master oscillator power amplifier (MOPA) configured transmitter, and a shared telescope transmitter and receiver to achieve opto-mechanically stable, and eye-safe, operation. A multistage filtered receiver is implemented to allow continuous measurement in high background conditions (e.g., bright daytime clouds).

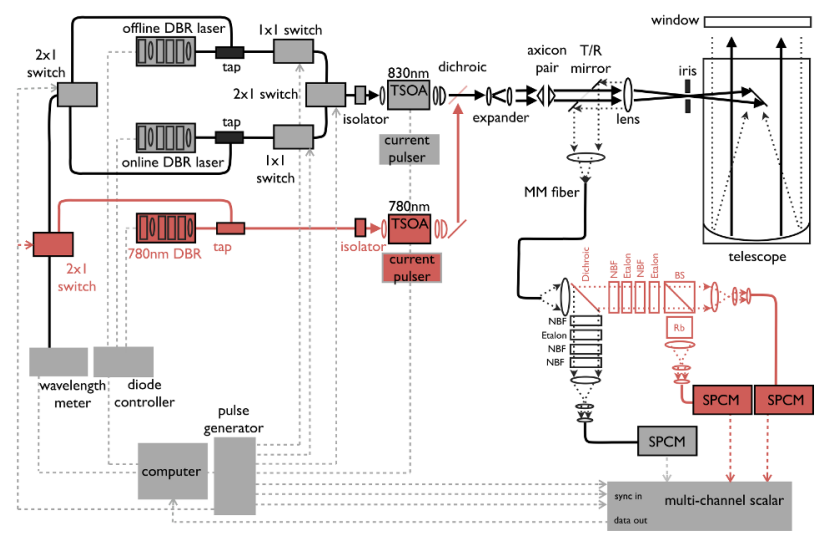

Figure 1. Schematic of the MPD system. One of the five units has been integrated with an HSRL channel (shown in red). $\mathrm{BS}=$ beam-splitter; $\mathrm{T} / \mathrm{R}=$ transmit receive; $\mathrm{Rb}=$ Rubidium vapor cell; $\mathrm{SPCM}=$ Single Photon Counting Module, NBF = narrow-band filter.

A pair of distributed Bragg reflector (DBR) semiconductor lasers nominally centered at $828 \mathrm{~nm}$ are interleaved at $100 \mathrm{~Hz}$ and used to seed a tapered semiconductor optical amplifier (TSOA). Current pulses to the TSOA amplify the laser and produce approximately $5 \mu \mathrm{J}$ over $900 \mathrm{~ns}$ duration. The beam is shaped into an annular ring with an axicon pair and then expanded to fill the inner part of the telescope with a radius of $10 \mathrm{~cm}$. The laser repetition rate, set to $7 \mathrm{kHz}$, limits the maximum altitude of the lidar system to $21 \mathrm{~km}$. Direct exposure to the laser exiting the telescope is within eye-safe limits. The backscattered light received is directed into a $105 \mu \mathrm{m}$ core multimode fiber in the receiver system. The light from the fiber is collimated and passes through a pair of interference filters and a solid etalon, with a combined passband of $0.014 \mathrm{~nm}$ for daylight rejection. The filtered light is then directed into a fiber coupled single photon counting module (SCPM), which utilizes a silicon avalanche photodiode operated in 'Geiger-mode'.

A diode-laser-based high spectral resolution lidar (HSRL) channel - based on the same low-cost lidar ar- 
chitecture [10] - was incorporated into one of the MPD units. A schematic of the combined system is shown in Figure 1. The upgraded instrument combines water vapor with quantitative measurements of aerosols and could significantly contribute to studies of aerosol-cloud interaction. Since HSRL do not require a correction for the geometric overlap function to obtain the aerosol backscatter coefficient, they are well suited to quantitative retrievals at comparatively low altitudes. As part of the network deployment, the diode-laser-based HSRL offers opportunities for higher resolution measurements of boundary layer height for model and satellite comparison studies as well as for continuous monitoring of this key observational parameter for weather and climate. Furthermore, to reduce alignment time at the start of field deployments, a fiber coupled transmitter package is being tested for these systems. This effort builds on a NASA funded SBIR which developed fiber-coupled isolated DBR lasers sources [11].

Following the completion of each water vapor MPD unit, they were operated continuously to test basic system functionality. For MPD unit \#3, this initial test period was approximately 6 months starting in the summer of 2018 as shown in Fig. 2. This initial test, done for all of the MPD units, demonstrated a high continuity time series in all weather and provided profiles of water vapor including periods of bright daytime clouds - from $300 \mathrm{~m}$ above ground level to $4-6 \mathrm{~km}$ (or cloud base, whichever is lower) with $150 \mathrm{~m}$ nominal vertical resolution and 1-5 minute temporal resolution. It is important to note, that this 6 month dataset was autonomously obtained - without human intervention - unless a subsystem was being upgraded as part of scheduled development and testing.

\subsection{Multi-Channel Scaler (MCS)}

A new MCS was developed for the testbed units to increase overall functionality, simplify the system (i.e., reduce part count), and improve the system integration reliability. The MCS used in the original prototype was a commercially available product from Sigma Space Inc., which required a proprietary software package that was then wrapped by another software package (in our case, Labview). The new MCS uses no proprietary software, and has advanced features while reducing overall system part counts. First, the MCS uses software defined switches local to the system's field-programmable gate array (FPGA). This feature allowed removal of the radio-frequency $(\mathrm{RF})$ hardware switches, and associated wiring, used in the prototype to route the signals from the photon counting module to the MCS. Second, the MCS has power monitoring channels, which allows for independent monitoring of rapidly switched laser pulses (e.g., both online and offline channels) via the FPGA software switches. This was not possible with the original hardware configuration and simplifies the system design by allowing removal of the third party power monitoring components and associated software. The gigabit Ethernet speed and software integration simplicity is a major advantage over the original system design.

The MCS includes eight input channels each capable of $90 \times 10^{6}$ pulse counts per second. Pulses are accumulated into range histograms that support 10 nanosecond to 20 microsecond bin sizes $(\approx 1.5$ meters to 3 kilometers in two-way distance), up to 3,000 bins, and as many as 30,000 profile accumulations. Complete histograms are output at rates as fast as $10 \mathrm{~Hz}$. Pulses received on a given input channel may be digitally and synchronously route-switched to different histogram accumulation channels, enabling the separation of online and offline wavelength accumulations without the need for external RF switches. Three separate synchronization inputs may be used to synchronize the start of histogram profile accumulations. Built-in data and synchronization test pattern options aid in system integration and development.

The MCS electronics are composed of a PicoZed processing module card and custom carrier card that includes pulse power detector channels, input buffering, power conditioning, and connectorization. The PicoZed processing card - a low-cost, off-the-shelf component developed by AVNET - includes a Xilinx Zynq System-onChip (SoC), 1GB memory, Ethernet and USB physical layer interfaces. The application utilizes less than $10 \%$

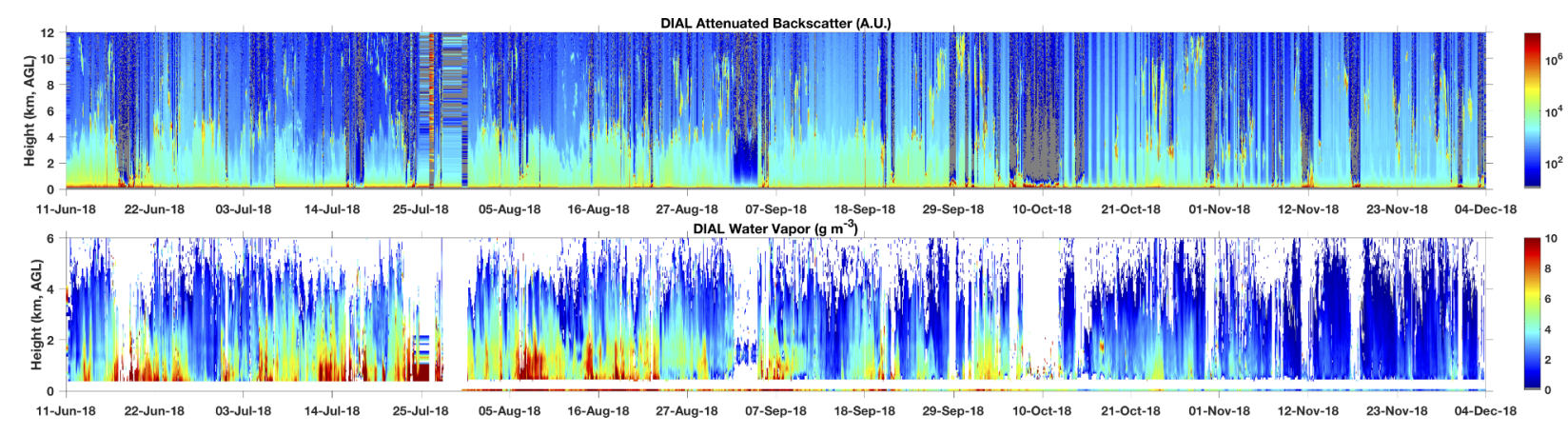

Figure 2. Time series of decimated 5-min 150-m MPD unit 3 profiles showing (top) range-corrected relative backscatter and (bottom) absolute humidity $\left(\mathrm{gm}^{-3}\right)$. Vertical white bars signify missing data due to MPD downtime from instrument testing periods (i.e., not hardware related) or attenuated signal above optically thick clouds. 
of available SoC's FPGA resources while operating at a $200 \mathrm{MHz}$ core clock rate, and the core processor readily handles the histogram data and metadata flow with its $600 \mathrm{MHz}$ CPU clock and direct memory access (DMA) routing to either the Gigabit Ethernet or USB2.0 interfaces. The MCS case occupies only the front half of a US-standard 19 inch rack 1 U slot. Pulse power detector channels utilize a filtering plus threshold detection technique to translate the amplitude of a pulse - with known pulse duration - into a time difference value that is measured via an FPGA input and simple timer counter. The time-based power measurement concept was adopted from the technique used by the University of Wisconsin lidar group.

\subsection{Data Acquisition and Processing Software}

The acquisition software of the MPD systems was completed with the following main goals: increasing fault tolerance, allowing flexibility to facilitate future system expansion, improved data quality and overall system duty cycle, and increased runtime stability. Most of these goals were achieved by altering the prototype operational structure of the acquisition software to run in parallel instead of a serial manner. Fault tolerance was achieved by isolating system critical components (e.g., the MCS control and laser control) from less critical components (e.g, housekeeping and real time data display). More monitoring resources are directed towards system critical components. Flexibility is further achieved using parallel control by isolating components that are expected to change with instrument modifications. An example of this is the number of lasers controlled. The water vapor DIAL profiling technique requires two laser transmitters (an online and an offline wavelength) but a third transmitter (for an HSRL) was added to unit two. Planned future upgrades to all of these systems (e.g., to measure temperature) will require additional lasers transmitters. Therefore, for the code to smoothly handle these hardware differences, the new architecture allows for additional controllers without changing any source code, just software controlled options. Data acquisition duty cycle is also improved by operating the control components in parallel, more completely utilizing computer resources. The combination of all of these changes allows for improved runtime stability without any loss of data quality. Furthermore, this combination of changes enhanced flexibility to accommodate software or hardware failures in the field or further development. Further modifications to the acquisition software beyond the overall structure includes the addition of more metadata for operational monitoring while deployed to field sites and the simplification of the metadata available. This adds redundancy for field operations. Calibration data and metadata such as that provided by the uninterruptible power supply were also added.

The post processing software used to verify system operation and performance was also heavily modified from the original prototype. The processing software was modified to accept changes to the acquisition software described above as well as adding necessary abstraction to facilitate future hardware changes. As a bonus of enhanced abstraction, simplification of the post processing software was achieved. Finally, minor changes were made to the archiving system hosted on NCAR servers to accommodate expansion from a single lidar unit to five.

\subsection{Environmental Housing}

The MPD testbed units were built using an aluminum frame that integrates the transceiver, custom telescope, and instrument rack. This aluminum frame is mounted to a shock and vibration isolation system, which helps minimize misalignment and potential damage during shipping. The housing optical window for each unit has a heating unit to help keep it clear during most operating conditions. Modifications from the initial prototype design are listed in Table 1 and bullet items below. The interior volume of the new system is $83 \%$ of the previous design, with reduced height (to makes transportation easier and simplify setup), and utilizes the same HVAC system to provide temperature stability over a wider range of environmental conditions.

Table 1. Interior dimensions of environmental enclosures

\begin{tabular}{ccc}
\hline & Unit 1 & Units $2-5$ \\
Width & $1.88 \mathrm{~m}(74 \mathrm{in})$ & $1.80 \mathrm{~m}(71 \mathrm{in})$ \\
Depth & $0.86 \mathrm{~m}(34 \mathrm{in})$ & $0.86 \mathrm{~m}(34 \mathrm{in})$ \\
Height & $1.73 \mathrm{~m}(68 \mathrm{in})$ & $1.49 \mathrm{~m}(58.5 \mathrm{in})$ \\
Volume & $2.80 \mathrm{~m}^{3}\left(99 \mathrm{ft}^{3}\right)$ & $2.32 \mathrm{~m}^{3}\left(82 \mathrm{ft}^{3}\right)$ \\
\hline
\end{tabular}

- Telescope redesigned for improved stability, ease of alignment, and reduced weight (e.g., $93 \mathrm{~kg}$ telescope assembly weight used in unit 1 was reduced to $54 \mathrm{~kg}$ in units 2-5.)

- Shock and vibration isolation system redesigned

- Heated window cell redesigned for lower fabrication costs

- Volume of container decreased (detailed in Table 1 above)

- Quick Pin mounted caster/wheels for easy removal

- Quick Pin mounted mast for weather station and cell modem

- Changed HVAC thermostat for tighter temperature control (tightening control band by $90 \%$ )

\section{FUTURE WORK}

As part of its 6-month commissioning phase, the five-unit testbed network is scheduled to be field tested from Apr to Jul 2019 at the Atmospheric Radiation Measurement (ARM) Southern Great Plains (SGP) atmospheric observatory in Oklahoma, USA. The test - conducted during a convectively active period, and performed alongside radiosondes, Raman lidar, and passive remote sensors for validation - is expected to provide valuable data to the science community. It is hoped that this initial demonstration of the technology will lead to fundamental advances within both the climate and weather forecasting communities, as it will allow observations of the 
evolution of the vertically-resolved thermodynamic environment at high temporal resolution for the first time. Furthermore, the evaluation of a test MPD network will be valuable in providing understanding if deployment of MPD type devices at a national scale could provide enough additional information to improve weather forecasts.

The complete five-unit MPD testbed is anticipated to be ready for service in late 2019 , and will be made available to the larger research community for field campaigns and deployment through NCAR. As the units were built with modular hardware and software - we are planning to integrate the testbed units with upgrades; such as HSRL channels and $\mathrm{O}_{2}$ :DIAL channels for temperature retrievals.

\section{SUMMARY}

A small testbed network of validated micro-pulse water vapor DIAL instruments has been completed. The diodelaser-based transmitter is inherently low-maintenance, low-cost, and designed to be eye-safe at the exit port. Each node provides continuous profiles of water vapor with complete daytime coverage - including periods of bright clouds - from $300 \mathrm{~m}$ above ground level to 4-6 km (or cloud base, whichever is lower) with $150 \mathrm{~m}$ nominal vertical resolution and 1 to 5 minute temporal resolution. A low-cost HSRL channel has been integrated into one of the instruments. The testbed is currently scheduled to be fielded at the ARM SGP site from Apr to Jul 2019. The test will be a significant step towards improving our understanding of the distribution of atmospheric water vapor, and provide the opportunity to evaluate the impact potential of a national scale network.

\section{ACKNOWLEDGMENTS}

NCAR is sponsored by the National Science Foundation. The authors would like to acknowledge the support of the National Science Foundation grant number 1624736.

\section{REFERENCES}

[1] National Research Council, 2009: Observing Weather and Climate from the Ground Up: A Nationwide Network of Networks. The National Academies Press, Washington, DC.

[2] National Research Council, 2010: When Weather Matters: Science and Service to Meet Critical Societal Needs. The National Academies Press, Washington, DC.

[3] National Research Council, 2012: Weather Services for the Nation: Becoming Second to None. The National Academies Press, Washington, DC.

[4] Wulfmeyer, V., R. M. Hardesty, D. D. Turner, A. Behrendt, M. P. Cadeddu, P. Di Girolamo, P. Schlssel, J. Van Baelen, and F. Zus, 2015: A review of the remote sensing of lower tropospheric thermodynamic profiles and its indispensable role for the understanding and the simulation of water and energy cycles Rev. Geophys., 53, 819-895.
[5] Nehrir, A. R., Repasky, K. S., Carlsten, J. L., Obland, M. D., and Shaw, J. A., 2009: Water Vapor Profiling Using a Widely Tunable, Amplified Diode-Laser-Based Differential Absorption Lidar (DIAL). Journal of Atmospheric and Oceanic Technology, 26(4), 733-745.

[6] Nehrir, A. R., Repasky, K. S., and Carlsten, J. L., 2011: Eye-Safe Diode-Laser-Based Micropulse Differential Absorption Lidar (DIAL) for Water Vapor Profiling in the Lower Troposphere. Journal of Atmospheric and Oceanic Technology, 28(2), 131147.

[7] Nehrir, A. R., Repasky, K. S., and Carlsten, J. L., 2012: Micropulse water vapor differential absorption lidar: transmitter design and performance. $O p$ tics express, 20(22), 25137-51.

[8] Spuler, S. M., Repasky, K. S., Morley, B., Moen, D., Hayman, M., and Nehrir, A. R., 2015: Field deployable diode-laser-based differential absorption lidar (DIAL) for profiling water vapor. Atmospheric Measurement Techniques, 8, 1073-1087.

[9] Weckwerth, T. M., K. Weber, D. D. Turner, and S. M. Spuler, 2016: Validation of a Water Vapor Micropulse Differential Absorption Lidar (DIAL). J. Atmospheric and Oceanic Technology, 33, 23532372.

[10] Hayman, M. and S. Spuler, 2017: Demonstration of a diode-laser-based high spectral resolution lidar (HSRL) for quantitative profiling of clouds and aerosols. Optics Express, 24, A1096.

[11] Overton G, 2016: Lidar: Differential absorption lidar demands high-power, narrowband DBR sources Laser Focus World, 52(9), 14-15. 